EPJ Web of Conferences 23, 00020 (2012)

DOI: $10.1051 /$ epjconf/20122300020

(C) Owned by the authors, published by EDP Sciences, 2012

\title{
Scanning Tunneling Microscopy Studies of Topological Insulators Grown by Molecular Beam Epitaxy
}

\author{
Xi Chen ${ }^{1}, \mathrm{Ke} \mathrm{He}^{2}$, Xucun $\mathrm{Ma}^{2}$, and Qikun Xue ${ }^{1,2}$ \\ ${ }^{1}$ State Key Laboratory of Low-Dimensional Quantum Physics and Department of Physics, Tsinghua University, Beijing \\ 100084, China \\ ${ }^{2}$ State Key Laboratory for Surface Physics, Institute of Physics, Chinese Academy of Sciences, Beijing 100190, China
}

\begin{abstract}
We summarize our recent scanning tunneling microscopy (STM) study of topological insulator thin films grown by molecular beam epitaxy (MBE), which includes the observation of electron standing waves on topological insulator surface and the Landau quantization of topological surface states. The work has provided valuable information to the understanding of intriguing properties of topological insulators, as predicted by theory.
\end{abstract}

\section{Introduction ${ }^{\mathrm{a}}$}

Topological insulator is a new class of quantum material, which possesses an energy gap in the bulk and nontrivial gapless states on the surface. The surface states are comprised of an odd number of massless Dirac cones in which crossing of two dispersion branches with opposite spins is fully protected by the time-reversal-symmetry at the Dirac point (figure 1). Topological insulators may provide new route to generating novel phases and quasiparticles, and may find applications in spintronics and quantum computing [1-7].

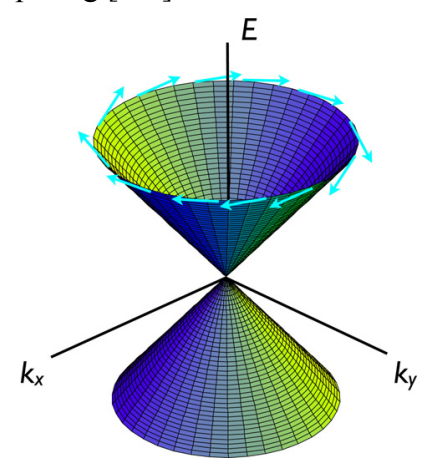

Fig. 1. Single Dirac cone band structure of topological insulators

In 2009, a class of binary compounds [8-11], $\mathrm{Bi}_{2} \mathrm{Se}_{3}$, $\mathrm{Bi}_{2} \mathrm{Te}_{3}$ and $\mathrm{Sb}_{2} \mathrm{Te}_{3}$, was shown to be the third generation of topological insulators. Compared to $\mathrm{Bi}_{1-\mathrm{x}} \mathrm{Sb}_{\mathrm{x}} \quad(0.07<\mathrm{x}$ $<0.22$ ) alloy, the surface states of the $\mathrm{Bi}_{2} \mathrm{Se}_{3}$ family

\footnotetext{
${ }^{\mathrm{a}}$ For correspondence, contact: xc@mail.tsinghua.edu.cn
}

contain only a single Dirac cone, making it easier to be studied. Also, these materials are stoichiometric compounds and hence in principle can be prepared at higher purity and quality. Finally and perhaps most importantly for applications, $\mathrm{Bi}_{2} \mathrm{Se}_{3}$ has a larger band gap of approximately $0.36 \mathrm{eV}$. It implies that topological behavior can sustain at room temperature. The unique properties of $\mathrm{Bi}_{2} \mathrm{Se}_{3}$ family have been experimentally identified by, for example angle resolved photoemission spectroscopy (ARPES), scanning tunneling microscopy (STM) and transport measurements.

STM is a surface sensitive technique based on the concept of quantum tunneling. The atomically resolved structure of a surface can be obtained by monitoring the tunneling current in an STM. Besides its unprecedented capability to study surface topography, information on electronic structure at a given location of a sample can also be obtained. This type of measurement is called scanning tunneling spectroscopy (STS) and typically results in a plot of the local density of states (LDOS) as a function of energy of the sample. It has been widely used in the characterization of metals, semiconductors and superconductors. Applying it to study of topological surface states, many intriguing properties have recently been unveiled. In this paper, we give an overview on the recent progress of STM study of topological insulators.

\section{Electronic standing waves on topological insulators}

In a crystal, the electronic eigen-states are described by Bloch wave-functions $\psi_{k}(\boldsymbol{r})$ with energy $\varepsilon$ and wavevector $\boldsymbol{k}$. The dispersion relation $\varepsilon(\boldsymbol{k})$ is usually measured 
by ARPES. In contrast, STM, which is a real space imaging technique, cannot be used to directly measure $\varepsilon(\boldsymbol{k})$ in momentum space. When disorders are introduced into a sample, the change in potential leads to the elastic electron scattering of the incident wave with a wave vector $\boldsymbol{k}_{\boldsymbol{i}}$ into $\boldsymbol{k}_{\boldsymbol{f}}=\boldsymbol{k}_{\boldsymbol{i}}+\boldsymbol{q}$, with $\boldsymbol{k}_{\boldsymbol{i}}$ and $\boldsymbol{k}_{\boldsymbol{f}}$ being on the same constant-energy contour (CEC). The quantum interference between the initial and final states results in a standing wave pattern whose spatial period is given by $\lambda=2 \pi /|\boldsymbol{q}|$, which are often referred to as the "Friedel oscillations". The phenomena can be observed by STM as modulations of the differential tunneling conductance. When the STM images of standing waves are Fourier transformed, the scattering wave vector $\boldsymbol{q}$ becomes visible in the reciprocal space. This way, it simultaneously provides real-space and momentum-space information.

In 1991, Don Eigler's group and Avouris' group independently reported the observation of standing waves on a metal surface by STM $[12,13]$. Afterwards, standing waves were imaged successively on other metal (Ag, $\mathrm{Au}$ and $\mathrm{Be}$ ) and semiconductor surfaces, and even on high- $\mathrm{T}_{\mathrm{c}}$ superconductors and graphene. STM has become a powerful technique to study wave-functions, electron scattering processes, and dispersion relation of a material.

The surface states of topological insulators possess spin-polarized Dirac-cone structure and are protected by time-reversal symmetry. These unique characters are supposed to reflect in the scattering processes and can be explored by standing waves using STM.

In this work, stoichiometric $\mathrm{Bi}_{2} \mathrm{Te}_{3}$ thin films were prepared on single crystal substrate $\mathrm{Si}(111)$ by MBE [14]. The atomically flat morphology imaged by STM reveals high quality of our samples. The surface states of $\mathrm{Bi}_{2} \mathrm{Te}_{3}$ were investigated by STS, which gives a measure of the local density of states of electrons at energy eV. The differential conductance in the bulk insulating gap linearly depends on the bias and is attributed to the gapless surface states. According to the calculations, the topological states of $\mathrm{Bi}_{2} \mathrm{Te}_{3}$ form a single Dirac cone at the center ( $\bar{\Gamma}$ point) of the surface Brillouin zone (SBZ) and the surface states around the $\bar{\Gamma}$ point overlap in energy with the bulk valence band. For this reason, the Dirac point is invisible in STS.
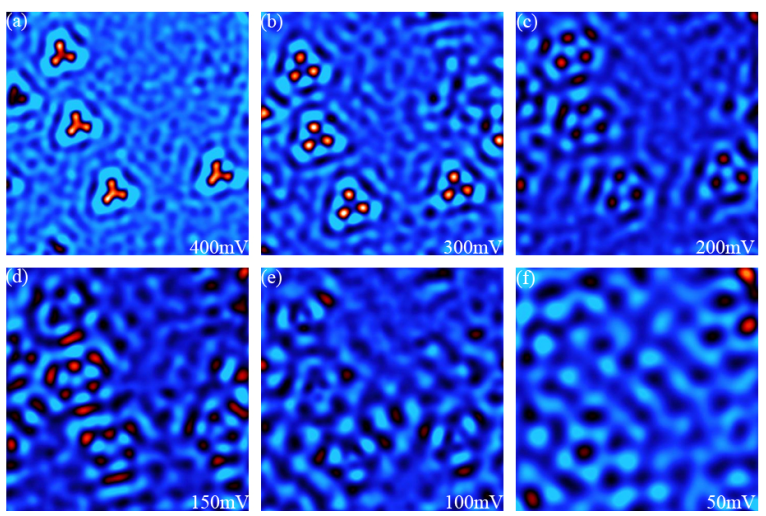

Fig. 2. (a) (f) Standing waves induced by Ag trimmers on $\mathrm{Bi}_{2} \mathrm{Te}_{3}(111)$ surface at various sample bias voltage. Each map is $28 \mathrm{~nm} \times 28 \mathrm{~nm}$ and has $128 \times 128$ pixels. The current was set at $0.1 \mathrm{nA}$.
We deposited a small amount of $\mathrm{Ag}$ atoms on the clean surface of the $\mathrm{Bi}_{2} \mathrm{Te}_{3}$ thin film. The atomically resolved STM image reveals that each Ag atom substitutes a $\mathrm{Bi}$ atom in the second layer [15]. The $\mathrm{dI} / \mathrm{dV}$ mapping was then carried out in a region containing $\mathrm{Ag}$ atoms. At each data point, the feedback was turned off and the bias modulation was turned on to record $\mathrm{dI} / \mathrm{dV}$. This procedure results in a series of spatial mapping of LDOS at various bias voltages.

Figures 2(a)-2(f) summarize the $\mathrm{dI} / \mathrm{dV}$ maps for bias voltages ranging from 50 to $400 \mathrm{mV}$. The first striking aspect of these images is the existence of standing waves in the vicinity of the $\mathrm{Ag}$ atoms. This phenomenon strongly supports the surface nature of topological states. For bulk states, there will be continuous ranges of wave vectors on the projected SBZ for a given energy. Usually, no distinct interference fringe can be produced by bulk states and visualized by STM.
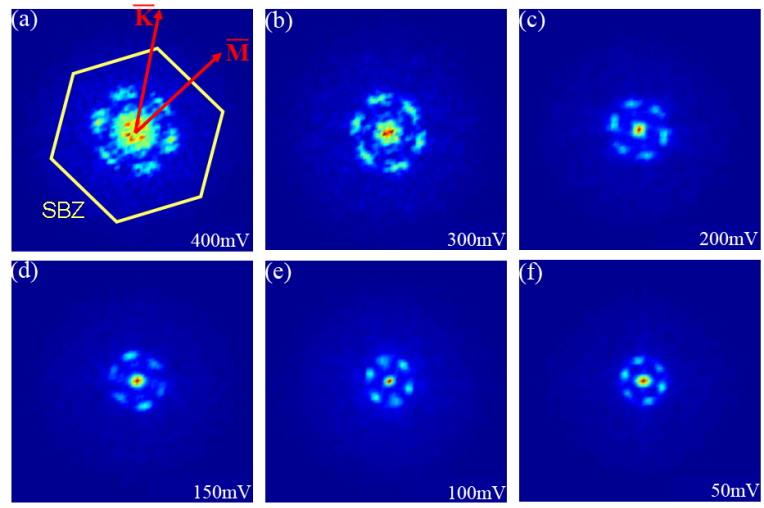

Fig. 3. (a) (f) The FFT power spectra of the $d I / d V$ maps in figure $2(\mathrm{a}) \sim(\mathrm{f})$. The SBZ in (a) is superimposed on the power spectra to indicate the directions in $\boldsymbol{q}$ space.

To quantify the standing waves and obtain the scattering wave vectors, we performed fast Fourier transformation (FFT) of the $\mathrm{dI} / \mathrm{dV}$ maps into the $\mathbf{q}$ space (figure 3). One important feature in the power spectra can be immediately discerned by comparing the six-fold symmetric pattern in the $\boldsymbol{q}$ space with the SBZ: the regions with high intensity are always oriented toward the $\bar{\Gamma}-\bar{M}$ directions, while the intensity in the $\bar{\Gamma}-\overline{\mathrm{K}}$ directions vanishes.

Such phenomena should be understood by exploring the standing wave formation mechanisms. In momentum space, $\boldsymbol{k}_{\boldsymbol{i}}$ and $\boldsymbol{k}_{\boldsymbol{f}}$ are on the same constant-energy contour (CEC) (figure 4). There are various directions of scattering between initial and final states. But most of these processes interfere destructively and cannot generate the observed standing waves. The interference patterns are dominated by the extremal points on the CEC, which resembles with Fermat's principle in optics. Applying the theory to $\mathrm{Bi}_{2} \mathrm{Te}_{3}$ with strong warping effects, two extremal points $\boldsymbol{q}_{1}$ and $\boldsymbol{q}_{2}$ are derived: $\boldsymbol{q}_{1}$ along $\bar{\Gamma}-\overline{\mathrm{K}}$ and $\boldsymbol{q}_{2}$ along $\bar{\Gamma}-\overline{\mathrm{M}}$.

However, only $\boldsymbol{q}_{2}$ generate the observed standing waves while $\boldsymbol{q}_{1}$ are invisible in the power spectra. There is a simple argument to account for the disappearance of $\boldsymbol{q}_{1}$ : the time-reversal invariance. $\boldsymbol{q}_{1}$ represents the 
backscattering between $\boldsymbol{k}$ and $-\boldsymbol{k}$. These two topological states are related by the time-reversal transformation: $|-\boldsymbol{k} \downarrow\rangle=\hat{T}|\boldsymbol{k} \uparrow\rangle$, where $\hat{T}$ is the time-reversal operator. It is straightforward to show that $\langle-\boldsymbol{k} \downarrow|\widehat{U}| \boldsymbol{k} \uparrow\rangle=$ $-\langle\boldsymbol{k} \uparrow|\widehat{U}|-\boldsymbol{k} \downarrow\rangle^{*}=-\langle-\boldsymbol{k} \downarrow|\widehat{U}| \boldsymbol{k} \uparrow\rangle=0$ for fermions, where $\widehat{U}$ represents the impurity potential of nonmagnetic $\mathrm{Ag}$ impurities and is a time-reversal invariant operator. Therefore, the backscattering between $\boldsymbol{k}$ and $-\boldsymbol{k}$ is quantum mechanically prohibited $[15,16]$. The absence of backscattering is a spectacular manifestation of the time-reversal symmetry, which offers a direct proof of the topological nature of the surface states.

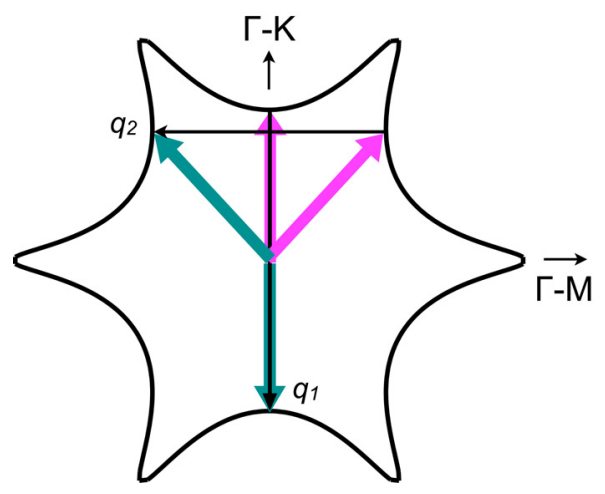

Fig. 4. The scattering geometry. The CEC is in the shape of a hexagram. The pink arrows indicate the initial wave-vector while the green ones indicate the incident wave-vector.

\section{Landau quantization of topological insulators}

Application of a magnetic field to conductors causes the charge carriers to circulate in cyclotron orbits with quantized energies called Landau levels (LLs). Landau quantization leads to characteristic oscillations in many properties such as magnetoresistance and it is also directly responsible for the well-known quantum Hall effect. In normal metals and two-dimensional electron gases, Landau levels are equally spaced. While for Dirac fermions, Landau levels consist of a field-independent state at the Dirac point and a sequence of levels with square-root dependence in both field and index, instead of the usual linear dependence:

$$
E_{n}=\operatorname{sgn}(n) \sqrt{2|n| \hbar e B v_{F}^{2}}, n=0, \pm 1, \pm 2 \cdots \text { (figure 5). }
$$

The energy $E_{n}$ is measured relative to the Dirac point.

STS was proved to be a powerful tool to study the Landau quantization in two-dimensional electron gas (2DEG) under magnetic fields. In 1998, Wiesendanger's group first observed the Landau levels by STS on nInAs(110) [17]. And later in 2005, Fukuyama's group observed landau levels on HOPG [18]. Remarkably, they obtained both the localized and extended states depending on energy relative to the Landau levels by using $\mathrm{dI} / \mathrm{dV}$ mappings near surface defects. The result provided a spectroscopic statement that HOPG has a high-mobility quasi-2DEG, which is eligible to show the quantum Hall effect. The first observation of Dirac fermion's Landau level was realized by Andrei's group of Rutgers University [19]. They found evidence of the coexistence of both massless and massive Dirac fermions in graphite, which is proposed to arise from different stacking structures of the graphite. After single-layer graphene was eventually isolated in 2004, it has become an ideal model system for theoretical and experimental study of Dirac fermions. On the surface of graphene grown on silicon carbide, Stroscio's group of NIST directly observed the non-equally-spaced energy spectrum of Landau levels, including the hallmark zeroenergy state of graphene which is independent of the applied magnetic field [20]. Notably, a complete linear collapse of the Landau level energies was obtained by plotting $E_{n}$ versus $(|n| B)^{1 / 2}$, confirming massless Dirac fermions in the epitaxial graphene.

Topological surface states also consist of the massless Dirac fermions as in graphene, so Landau quantization is predicted to be observed in this system. The $\mathrm{Bi}_{2} \mathrm{Se}_{3}$ film was epitaxially grown on graphitized $6 \mathrm{H}-\mathrm{SiC}(0001)$ substrate and has lower carrier density than those prepared by the self-flux technique, as demonstrated by previous works $[21,22]$. The STM images exhibit the hexagonal lattice structure and very small density of defects. The high quality of the MBE films ensures the observation of Landau levels.

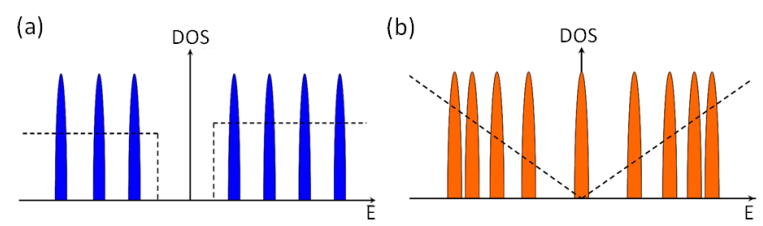

Fig. 5. Landau quantization of (a) 2D free electon gas and (b) Dirac fermions.

At zero magnetic fields, the surface states of $\mathrm{Bi}_{2} \mathrm{Se}_{3}$ were investigated by $\mathrm{dI} / \mathrm{dV}$ measurement [23]. The Dirac point of the topological states corresponds to the minimum of the spectrum. When magnetic field is applied perpendicular to the sample surface, a series of peaks of tunneling conductance are explicitly resolved above the Dirac point. The magnetic field dependence of these peaks in figure 6(a) clearly reveals the development of well-defined Landau levels in $\mathrm{Bi}_{2} \mathrm{Se}_{3}$ with increasing field. The spectra demonstrate a direct measurement of the Landau quantization of the topological surface states. It has some common features with that of graphene. Unlike the Landau levels of a 2DEG with parabolic dispersion, the peaks are not equally spaced. In addition, there is always one peak residing at the Dirac point in each $\mathrm{dI} / \mathrm{dV}$ spectrum. The energy of this $\mathrm{LL}$ is independent of the magnetic field. The absence of LLs below the Dirac point results from the overlapping of the surface states with the bulk valence band.

The Dirac fermion nature of the electrons is revealed by plotting the energies of LLs $v s .(|n| B)^{1 / 2}$. The peak positions of LLs are determined by fitting the differential 
conductance with multiple Gaussians. Notably, the $E_{n}$ 's in the vicinity of the Fermi level fall on a straight line as predicted. However, the energies of LLs with smaller index $\mathrm{n}$ deviate from the linear fitting. This deviation can be understood by considering the electrostatic field between the sample surface and the STM tip. The density of the induced charges in the presence of a tip is given by $\varepsilon_{0} \mathrm{~V} / \mathrm{d}$, where $\mathrm{V} \sim 100 \mathrm{mV}$ and $\mathrm{d} \sim 1 \mathrm{~nm}$ are the sample bias voltage and the distance between tip and sample, respectively. Typically, the induced density of electrons is in the order of $10^{12} \mathrm{~cm}^{-2}$, which is comparable to the intrinsic carrier density on the surface without a tip. Thus, the effect due to the electric field in tunneling junction is not negligible. With negative bias voltage, the field effect moves the Dirac cone downwards to accommodate the induced charges. Consequently, the apparent positions of the Landau levels with small $\mathrm{n}$ tend to stay above the linear relation. After a calibration was performed to the energy of Landau levels to eliminate this electrostatic field, all the data collapse to a single line, suggesting that the energy of LLs is scaled as $(|n| B)^{1 / 2}$ (figure 6(b)). Fitting the data to a straight line gives $v_{F}=3.4 \times 10^{5} \mathrm{~m} / \mathrm{s}$.
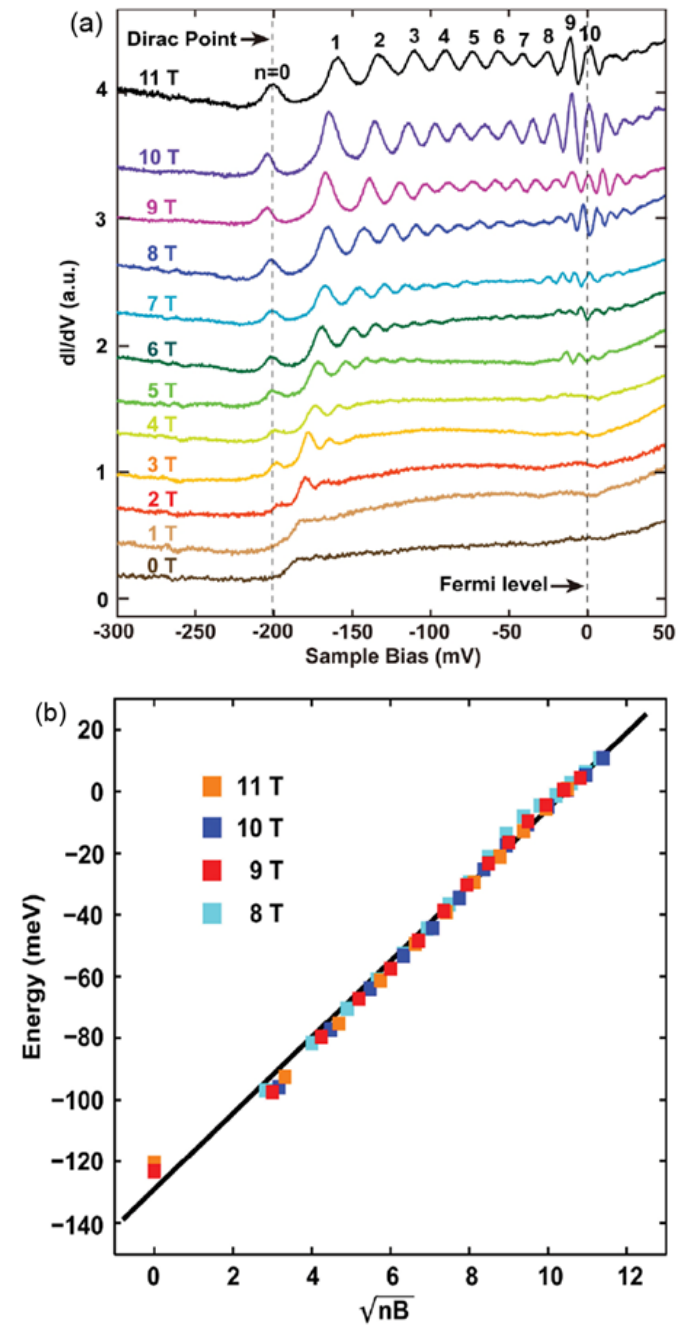

Fig. 6. (a) Landau quantization of $\mathrm{Bi}_{2} \mathrm{Se}_{3}$ film acquired for various magnetic fields from $0 \mathrm{~T}$ to $11 \mathrm{~T}$ (b) The fitting to the $\mathrm{LL}$ energies for magnetic fields from $8 \mathrm{~T}$ to $11 \mathrm{~T}$.
We further confirm the 2D nature of the quantized states by evaporating $\mathrm{Ag}$ atoms onto the surface of $\mathrm{Bi}_{2} \mathrm{Se}_{3}$. Presumably, the LLs are sensitive to the defect scattering if the impurities are located within the 2DEG. At low defect density, no explicit change in the tunneling spectrum has been observed (figure 7(a)). The defect scattering comes into effect when the distance between impurities in the 2DEG is comparable to the magnetic length (figure 7(b)). Finally, at even higher $\mathrm{Ag}$ atom coverage, the Landau quantization is completely suppressed as expected (figure 7(c)). The presence of distinct peaks and the suppression of LLs by surface impurities strongly indicate that the peaks are due to Landau quantization of the topological surface states within the bulk gap of $\mathrm{Bi}_{2} \mathrm{Se}_{3}$. The observation of Landau quantization may eventually lead to the realization of quantum Hall effect in topological insulators.

(a)
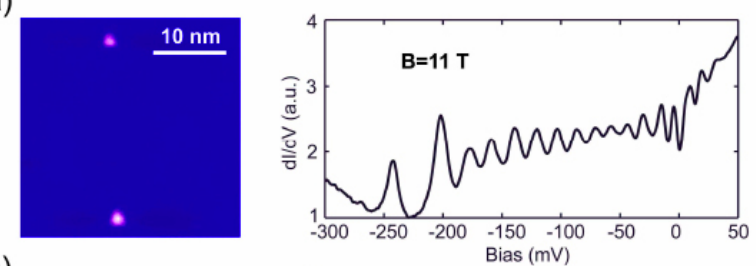

(b)
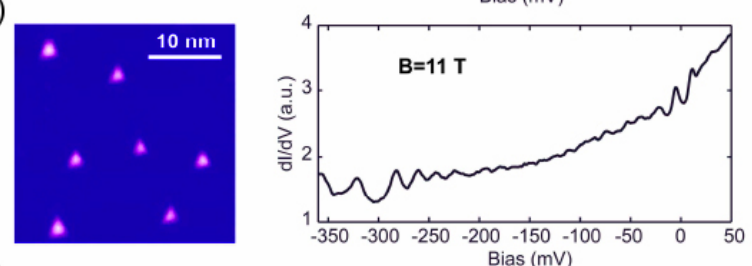

(c)
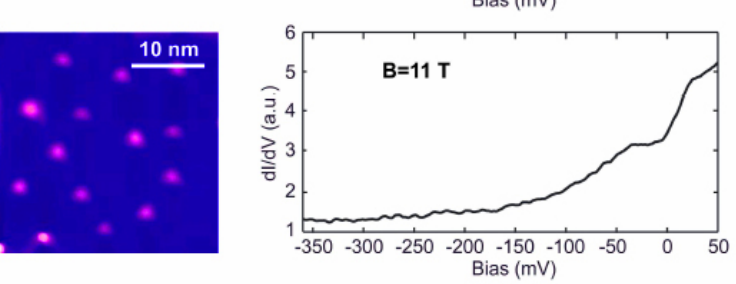

Fig. 7. Suppression of LLs by defects. The three panels show the STM images and the corresponding STS spectra of LLs at different $\mathrm{Ag}$ densities, respectively.

\section{Conclusion}

The field of topological insulators is growing rapidly. The basic properties have been established by theoretical insight and experimental discoveries. However, there is still much work to be done to realize the potential application of these dramatic systems. Combining topological insulators with ordinary superconductors or magnetic materials is predicted to give rise to exotic particles or states, such as topological magnetoelectric effect [24] and Majorana fermions [25, 26]. These predictions put forward great challenges to materials synthesis and experimental measurements. STM is expected to play more important role in this exciting direction. 


\section{References}

1. C. L. Kane and E. J. Mele, Phys. Rev. Lett. 95, 226801 (2005).

2. B. A. Bernevig, T. L. Hughes and S. C. Zhang Science 314, 1757 (2006).

3. KÄonig M, Wiedmann S, Brune $\mathrm{C}$ et al. Quantum Spin Hall Insulator State in HgTe Quantum Wells. Science 318 (2007) 766-770.

4. L. Fu, C. L. Kane and E. J. Mele, Phys. Rev. Lett. 98, 106803 (2007).

5. L. Fu and C. L. Kane, Phys. Rev. B 76, 045302 (2007).

6. D. Hsieh, D. Qian, L. Wray et al., Nature 452, 970 (2008).

7. D. Hsieh, Y. Xia, L. Wray et al., Science 323, 919 (2009).

8. H. J. Zhang, C. X. Liu, X. L. Qi et al., Nat. Phys. 5, 438 (2009).

9. J. Moore, Nat. Phys. 5, 378 (2009).

10. Y. Xia, D. Qian, D. Hsieh et al., Nat. Phys. 5, 398 (2009).

11. Y. L. Chen, J. G. Analytis and J. H. Chu, Science 325, 178 (2009).

12. M. F. Crommie, C. P. Lutz and D. M. Eigler, Nature 363, 524 (1993).

13. Y. Hasegawa and Ph. Avouris, Phys. Rev. Lett. 71, 1071 (1993).

14. Y. Y. Li, G. Wang, X. G. Zhu et al., Adv. Mater. 22, 4002 (2010).

15. T. Zhang, P. Cheng, X. Chen et al., Phys. Rev. Lett. 103, 266803 (2009).

16. W. C. Lee, C. J. Wu, D. P. Arovas et al., Phys. Rev. B 80, 245439 (2009).

17. R. Dombrowski, Chr. Wittneven, M. Morgenstern and R. Wiesendanger, Appl. Phys. A 66, S203 (1998).

18. T. Matsui, H. Kambara, Y. Niimi, K. Tagami, M. Tsukada and H. Fukuyama, Phys. Rev. Lett. 94, 226403 (2005).

19. G. H. Li and E. Y. Andrei, Nat. Phys. 3, 623 (2007).

20. Miller D L, Kubista K D, Rutter G M et al. Observing the Quantization of Zero Mass Carriers in Graphene. Science 324(2009) 924-927

21. Song C L, Wang Y L, Jiang Y P et al. Topological insulator $\mathrm{Bi}_{2} \mathrm{Se}_{3}$ thin films grown on double-layer graphene by molecular beam epitaxy. Appl. Phys. Lett. 97(2010) 143118-143121

22. Y. Zhang, K. He, C. Z. Chang et al., Nat. Phys. 6, 584 (2010).

23. P. Cheng, C. L. Song, T. Zhang et al., Phys. Rev. Lett. 105, 076801 (2010).

24. X. L. Qi, R. Li, J. D. Zang et al., Science 323, 1184 (2009).

25. L. Fu and C. L. Kane, Phys. Rev. Lett. 100, 096407 (2008).

26. J. C. Y. Teo and C. L. Kane, Phys. Rev. Lett. 104, 046401 (2010). 